\title{
Evaluating a Focus-Based Approach to Anaphora Resolution*
}

\author{
Saliha Azzam, Kevin Humphreys and Robert Gaizauskas \\ \{s.azzam, k. humphreys, r.gaizauskas\}@dcs. shef .ac.uk \\ Department of Computer Science, University of Sheffield \\ Regent Court, Portobello Road \\ Sheffield S1 4DP UK
}

\begin{abstract}
We present an approach to anaphora resolution based on a focusing algorithm, and implemented within an existing MUC (Message Understanding Conference) Information Extraction system, allowing quantitative evaluation against a substantial corpus of annotated real-world texts. Extensions to the basic focusing mechanism can be easily tested, resulting in refinements to the mechanism and resolution rules. Results show that the focusing algorithm is highly sensitive to the quality of syntactic-semantic analyses, when compared to a simpler heuristic-based approach.
\end{abstract}

\section{Introduction}

Anaphora resolution is still present as a significant linguistic problem, both theoretically and practically, and interest has recently been renewed with the introduction of a quantitative evaluation regime as part of the Message Understanding Conference (MUC) evaluations of Information Extraction (IE) systems (Grishman and Sundheim, 1996). This has made it possible to evaluate different (implementable) theoretical approaches against sizable corpora of real-world texts, rather than the small collections of artificial examples typically discussed in the literature.

This paper describes an evaluation of a focusbased approach to pronoun resolution (not anaphora in general), based on an extension of Sidner's algorithm (Sidner, 1981) proposed in (Azzam, 1996), with further refinements from development on real-world texts. The approach

\footnotetext{
* This work was carried out in the context of the EU AVENTINUS project (Thumair, 1996), which aims to develop a multilingual IE system for drug enforcement, and including a language-independent coreference mechanism (Azzam et al., 1998).
}

is implemented within the general coreference mechanism provided by the LaSIE (Large Scale Information Extraction) system (Gaizauskas et al., 1995) and (Humphreys et al., 1998), Sheffield University's entry in the MUC-6 and 7 evaluations.

\section{Focus in Anaphora Resolution}

The term focus, along with its many relations such as theme, topic, center, etc., reflects an intuitive notion that utterances in discourse are usually 'about' something. This notion has been put to use in accounts of numerous linguistic phenomena, but it has rarely been given a firm enough definition to allow its use to be evaluated. For anaphora resolution, however, stemming from Sidner's work, focus has been given an algorithmic definition and a set of rules for its application. Sidner's approach is based on the claim that anaphora generally refer to the current discourse focus, and so modelling changes in focus through a discourse will allow the identification of antecedents.

The algorithm makes use of several focus registers to represent the current state of a discourse: $C F$, the current focus; $A F L$, the alternate focus list, containing other candidate foci; and $F S$, the focus stack. A parallel structure to the $C F, A F$ the actor focus, is also set to deal with agentive pronouns. The algorithm updates these registers after each sentence, confirming or rejecting the current focus. A set of Interpretation Rules (IRs) applies whenever an anaphor is encountered, proposing potential antecedents from the registers, from which one is chosen using other criteria: syntactic, semantic, inferential, etc. 


\subsection{Evaluating Focus-Based Approaches}

Sidner's algorithmic account, although not exhaustively specified, has lead to the implementation of focus-based approaches to anaphora resolution in several systems, e.g. PIE (Lin, 1995). However, evaluation of the approach has mainly consisted of manual analyses of small sets of problematic cases mentioned in the literature. Precise evaluation over sizable corpora of real-world texts has only recently become possible, through the resources provided as part of the MUC evaluations.

\section{Coreference in LaSIE}

The LaSIE system (Gaizauskas et al., 1995) and (Humphreys et al., 1998), has been designed as a general purpose IE system which can conform to the MUC task specifications for named entity identification, coreference resolution, IE template element and relation identification, and the construction of scenario-specific IE templates. The system is basically a pipeline architecture consisting of tokenisation, sentence splitting, part-of-speech tagging, morphological stemming, list lookup, parsing with semantic interpretation, proper name matching, and discourse interpretation. The latter stage constructs a discourse model, based on a predefined domain model, using the, often partial, semantic analyses supplied by the parser.

The domain model represents a hierarchy of domain-relevant concept nodes, together with associated properties. It is expressed in the XI formalism (Gaizauskas, 1995) which provides a basic inheritance mechanism for property values and the ability to represent multiple classificatory dimensions in the hierarchy. Instances of concepts mentioned in a text are added to the domain model, populating it to become a text-, or discourse-, specific model.

Coreference resolution is carried out by attempting to merge each newly added instance, including pronouns, with instances already present in the model. The basic mechanism is to examine, for each new-old pair of instances: semantic type consistency/similarity in the concept hierarchy; attribute value consistency/similarity, and a set of heuristic rules, some specific to pronouns, which can act to rule out a proposed merge. These rules can refer to various lexical, syntactic, semantic, and po- sitional information about instances. The integration of the focus-based approach replaces the heuristic rules for pronouns, and represents the use of LaSIE as an evaluation platform for more theoretically motivated algorithms. It is possible to extend the approach to include definite NPs but, at present, the existing rules are retained for non-pronominal anaphora in the MUC coreference task: proper names, definite noun phrases and bare nouns.

\section{Implementing Focus-Based Pronoun Resolution in LaSIE}

Our implementation makes use of the algorithm proposed in (Azzam, 1996), where elementary events ( $E E \mathrm{~s}$, effectively simple clauses) are used as basic processing units, rather than sentences. Updating the focus registers and the application of interpretation rules (IRs) for pronoun resolution then takes place after each $E E$, permitting intrasentential references. ${ }^{1}$ In addition, an initial 'expected focus' is determined based on the first $E E$ in a text, providing a potential antecedent for any pronoun within the first $E E$.

Development of the algorithm using realworld texts resulted in various further refinements to the algorithm, in both the $I R$ s and the rules for updating the focus registers. The following sections describe the two rules sets separately, though they are highly interrelated in both development and processing.

\subsection{Updating the Focus}

The algorithm includes two new focus registers, in addition to those mentioned in section 2: $A F S$, the actor focus stack, used to record previous $A F$ (actor focus) values and so allow a separate set of $I R \mathrm{~s}$ for agent pronouns (animate verb subjects); and Intra- $A F L$, the intrasentential alternate focus list, used to record candidate foci from the current $E E$ only.

In the space available here, the algorithm is best described through an example showing the use of the registers. This example is taken from a New York Times article in the MUC-7 training corpus on aircraft crashes:

\footnotetext{
${ }^{1}$ An important limitation of Sidner's algorithm, noted in (Azzam, 1996), is that the focus registers are only updated after each sentence. Thus antecedents proposed for an anaphor in the current sentence will always be from the previous sentence or before and intrasentential references are impossible.
} 
State Police said witnesses told them the propeller was not turning as the plane descended quickly toward the highway in Wareham near Exit 2. It hit a tree.

\section{EE-1: State Police said tell_event}

An 'expected focus' algorithm applies to initialise the registers as follows:

$C F$ (current focus) $=$ tell_event

$A F$ (actor focus) $=$ State Police

Intra- $A F L$ remains empty because EE-1 contains no other candidate foci. No other registers are affected by the expected focus. No pronouns occur in EE-1 and so no IRs apply.

\section{EE-2: witnesses told them}

The Intra- $A F L$ is first initialised with all (non-pronominal) candidate foci in the EE:

Intra- $A F L=$ witnesses

The IRs are then applied to the first pronoun, them, and, in this case, propose the current $A F$, State Police, as the antecedent. The Intra-AFL is immediately updated to add the antecedent:

Intra-AFL $=$ State Police, witnesses

$\mathrm{EE}-2$ has a pronoun in 'thematic' position, 'theme' being either the object of a transitive verb, or the subject of an intransitive or the copula (following (Gruber, 1976)). Its antecedent therefore becomes the new $C F$, with the previous value moving to the $F S$. EE-2 has an 'agent', where this is an animate verb subject (again as in (Gruber, 1976)), and this becomes the new $A F$. Because the old $A F$ is now the $C F$, it is not added to the $A F S$ as it would be otherwise. After each $\mathrm{EE}$ the Intra-AFL is added to the current $A F L$, excluding the $C F$. The state after EE-2 is then:

$$
\begin{array}{ll}
C F=\text { State Police } & A F=\text { witnesses } \\
F S=\text { tell_event } & A F L=\text { witnesses }
\end{array}
$$

EE-3: the propeller was not turning

The Intra- $A F L$ is reinitialised with candidate foci from this EE:

\section{Intra- $A F L=$ propeller}

No pronouns occur in EE-3 and so no $I R \mathrm{~s}$ apply. The 'theme', propeller here because of the copula, becomes the new $C F$ and the old one is added to the $F S$. The $A F$ remains unchanged as the current $E E$ lacks an agent:

$C F=$ propeller

$A F=$ witnesses
$F S=$ State Police, tell_event

$A F L=$ propeller, witnesses

EE-4: the plane descended

Intra- $A F L=$ the plane

$C F=$ the plane (theme)

$A F=$ witnesses (unchanged)

$F S=$ propeller, State Police, tell_event

$A F L=$ the plane, propeller, witnesses

In the current algorithm the $A F L$ is reset at this point, because EE-4 ends the sentence.

EE-5: it hit a tree

Intra- $A F L=a$ tree

The $I R$ s resolve the pronoun it with the $C F$ :

$C F=$ the plane (unchanged)

$A F=$ witnesses (unchanged)

$F S=$ propeller, State Police, tell_event

$A F L=a$ tree

\subsection{Interpretation Rules}

Pronouns are divided into three classes, each with a distinct set of IRs proposing antecedents:

Personal pronouns acting as agents (animate subjects): (e.g. he in Shotz said he knew the pilots) $A F$ proposed initially, then animate members of $A F L$.

Non-agent pronouns: (e.g. them in EE-2 above and it in EE-5) $C F$ proposed initially, then members of the $A F L$ and $F S$.

Possessive, reciprocal and reflexive pronouns (PRRs): (e.g. their in the brothers had left and were on their way home) Antecedents proposed from the Intra- $A F L$, allowing intra-EE references.

Antecedents proposed by the $I R$ s are accepted or rejected based on their semantic type and feature compatibility, using the semantic and attribute value similarity scores of LaSIE's existing coreference mechanism.

\section{Evaluation with the MUC Corpora}

As part of MUC (Grishman and Sundheim, 1996), coreference resolution was evaluated as a sub-task of information extraction, which involved negotiating a definition of coreference relations that could be reliably evaluated. The final definition included only 'identity' relations between text strings: proper nouns, common nouns and pronouns. Other possible coreference relations, such as 'part-whole', and nontext strings (zero anaphora) were excluded. 
The definition was used to manually annotate several corpora of newswire texts, using SGML markup to indicate relations between text strings. Automatically annotated texts, produced by systems using the same markup scheme, were then compared with the manually annotated versions, using scoring software made available to MUC participants, based on (Vilain et al., 1995).

The scoring software calculates the standard Information Retrieval metrics of 'recall' and 'precision', ${ }^{2}$ together with an overall $f$-measure. The following section presents the results obtained using the corpora and scorer provided for MUC-7 training (60 texts, average 581 words per text, 19 words per sentence) and evaluation (20 texts, average 605 words per text, 20 words per sentence), the latter provided for the formal MUC-7 run and kept blind during development.

\section{Results}

The MUC scorer does not distinguish between different classes of anaphora (pronouns, definite noun phrases, bare nouns, and proper nouns), but baseline figures can be established by running the LaSIE system with no attempt made to resolve any pronouns:

\begin{tabular}{lrcc}
\multicolumn{1}{c}{ Corpus } & Recall & Precision & $f$ \\
Training: & $42.4 \%$ & $73.6 \%$ & $52.6 \%$ \\
Evaluation: & $44.7 \%$ & $73.9 \%$ & $55.7 \%$
\end{tabular}

LaSIE with the simple pronoun resolution heuristics of the non-focus-based mechanism achieves the following:

\begin{tabular}{lrcc}
\multicolumn{1}{c}{ Corpus } & Recall & Precision & $f$ \\
Training: & $58.2 \%$ & $71.3 \%$ & $64.1 \%$ \\
Evaluation: & $56.0 \%$ & $70.2 \%$ & $62.3 \%$
\end{tabular}

showing that more than three quarters of the estimated $20 \%$ of pronoun coreferences in the corpora are correctly resolved with only a minor loss of precision.

LaSIE with the focus-based algorithm achieves the following:

\footnotetext{
${ }^{2}$ Recall is a measure of how many correct (i.e. manually annotated) coreferences a system found, and precision is a measure of how many coreferences that the system proposed were actually correct. For example, with 100 manually annotated coreference relations in a corpus and a system that proposes 75 , of which 50 are correct, recall is then $50 / 100$ or $50 \%$ and precision is $50 / 75$ or $66.7 \%$.
}

\begin{tabular}{lrcc}
\multicolumn{1}{c}{ Corpus } & Recall & Precision & $\mathbf{f}$ \\
Training: & $55.4 \%$ & $70.3 \%$ & $61.9 \%$ \\
Evaluation: & $53.3 \%$ & $69.7 \%$ & $60.4 \%$
\end{tabular}

which, while demonstrating that the focusbased algorithm is applicable to real-world text, does question whether the more complex algorithm has any real advantage over LaSIE's original simple approach.

The lower performance of the focus-based algorithm is mainly due to an increased reliance on the accuracy and completeness of the grammatical structure identified by the parser. For example, the resolution of a pronoun will be skipped altogether if its role as a verb argument is missed by the parser. Partial parses will also affect the identification of $E E$ boundaries, on which the focus update rules depend. For example, if the parser fails to attach a prepositional phrase containing an antecedent, it will then be missed from the focus registers and so the IRs (see (Azzam, 1995)). The simple LaSIE approach, however, will be unaffected in this case.

Recall is also lost due to the more restricted proposal of candidate antecedents in the focusbased approach. The simple LaSIE approach proposes antecedents from each preceding paragraph until one is accepted, while the focusbased approach suggests a single fixed set.

From a theoretical point of view, many interesting issues appear with a large set of examples, discussed here only briefly because of lack of space. Firstly, the fundamental assumption of the focus-based approach, that the focus is favoured as an antecedent, does not always apply. For example:

In June, a few weeks before the crash of TWA Flight 800, leaders of several Middle Eastern terrorist organizations met in Teheran to plan terrorist acts. Among them was the PFL of Palestine, an organization that has been linked to airplane bombings in the past.

Here, the pronoun them corefers with organizations rather than the focus leaders. Additional information will be required to override the fundamental assumption.

Another significant question is when sentence focus changes. In our algorithm, focus changes when there is no reference (pronominal or otherwise) to the current focus in the current 
$E E$. In the example used in section 4.1 , this causes the focus at the end of the first sentence to be that of the last $E E$ in that sentence, thus allowing the pronoun it in the subsequent sentence to be correctly resolved with the plane. However in the example below, the focus of the first $E E$ (the writ) is the antecedent of the pronoun it in the subsequent sentence, rather than the focus from the last $E E$ (the ...fight):

The writ is for "damages" of seven passengers who died when the Airbus A310 fight crashed. It claims the deaths were caused by negligence.

Updating focus after the complete sentence, rather than each $E E$, would propose the correct antecedent in this case. However neither strategy has a significant overall advantage in our evaluations on the MUC corpora.

Another important factor is the priorities of the Interpretation Rules. For example, when a personal pronoun can corefer with both $C F$ and $A F, I R$ s select the $C F$ first in our algorithm. However, this priority is not fixed, being based only on the corpora used so far, which raises the possibility of automatically acquiring $I R$ priorities through training on other corpora.

\section{Conclusion}

A focus-based approach to pronoun resolution has been implemented within the LaSIE IE system and evaluated on real-world texts. The results show no significant preformance increase over a simpler heuristic-based approach. The main limitation of the focus-based approach is its reliance on a robust syntactic/semantic analysis to find the focus on which all the IRs depend. Examining performance on the realworld data also raises questions about the theoretical assumptions of focus-based approaches, in particular whether focus is always a favoured antecedent, or whether this depends, to some extent, on discourse style.

Analysing the differences in the results of the focus- and non-focus-based approaches, does show that the focus-based rules are commonly required when the simple syntactic and semantic rules propose a set of equivalent antecedents and can only select, say, the closest arbitrarily. A combined approach is therefore suggested, but whether this would be more effect- ive than further refining the resolution rules of the focus-based approach, or improving parse results and adding more detailed semantic constraints, remains an open question.

\section{References}

S. Azzam, K. Humphreys, and R. Gaizauskas. 1998. Coreference resolution in a multilingual information extraction system. In Proceedings of the First Language Resources and Evaluation Conference (LREC). Linguistic Coreference Workshop.

S. Azzam. 1995. Anaphors, PPs and Disambiguation Process for conceptual analysis. In Proceedings of 14th IJCAI.

S. Azzam. 1996. Resolving anaphors in embedded sentences. In Proceedings of 34th ACL.

R. Gaizauskas, T. Wakao, K Humphreys, H. Cunningham, and Y. Wilks. 1995. Description of the LaSIE system. In Proceedings of MUC-6, pages 207-220. Morgan Kaufmann.

R. Gaizauskas. 1995. XI: A Knowledge Representation Language Based on CrossClassification and Inheritance. Technical Report CS-95-24, University of Sheffield.

R. Grishman and B. Sundheim. 1996. Message Understanding Conference - 6: A brief history. In Proceedings of 16th IJCAI, pages 466-471.

J.S. Gruber. 1976. Lexical structures in syntax and semantics. North-Holland.

K. Humphreys, R. Gaizauskas, S. Azzam, C. Huyck, B. Mitchell, H. Cunningham, and Y. Wilks. 1998. Description of the LaSIE-II system. In Proceedings of $M U C-\gamma$. Forthcoming.

D. Lin. 1995. Description of the PIE System. In Proceedings of $M U C-6$, pages 113-126. Morgan Kaufmann.

C. Sidner. 1981. Focusing for interpretation of pronouns. American Journal of Computational Linguistics, 7:217-231.

G. Thurmair. 1996. AVENTINUS System Architecture. AVENTINUS project report LE12238.

M. Vilain, J. Burger, J. Aberdeen, D. Connolly, and L. Hirschman. 1995. A model-theoretic coreference scoring scheme. In Proceedings of MUC-6, pages 45-52. Morgan Kaufmann. 